AsClepIO. Revista de Historia de la Medicina y de la Ciencia

65 (2), julio-diciembre 2013, p019

ISSN-L:0210-4466

http://dx.doi.org/10.3989/asclepio.2013.19

ESTUDIOS / RESEARCH STUDIES

\title{
APORTACIONES AL CÁLCULO DE LA LATITUD EN LA NÁUTICA ESPAÑOLA \\ DE PRINCIPIOS DEL SIGLO XVII CONTENIDAS EN EL MANUSCRITO DE DIEGO RAMÍREZ DE ARELLANO RECONOCIMIENTO DE LOS ESTRECHOS DE MAGALLANES Y SAN VICENTE, CON ALGUNAS COSAS CURIOSAS DE NAVEGACIÓN (1621)
}

\author{
Ignacio Díaz Hernández \\ Universidad de Valencia \\ ignacio-díaz@telefonica.net
}

Recibido: 25 de mayo de 2011; Aceptado: 28 de febrero de 2013

Cómo citar este artículo/Citation: Díaz Hernández, Ignacio (2013), “Aportaciones al cálculo de la latitud en la náutica española de principios del siglo XVII contenidas en el manuscrito de Diego Ramírez de Arellano Reconocimiento de los estrechos de Magallanes y San Vicente, con algunas cosas curiosas de navegación (1621)", Asclepio 65 (2): p019. doi: http://dx.doi.org/10.3989/asclepio.2013.19

RESUMEN: En 1620 es nombrado piloto mayor de la Casa de Contratación de Sevilla Diego Ramírez de Arellano, autor de un manuscrito titulado Reconocimiento de los estrechos de Magallanes y San Vicente, con algunas cosas curiosas de navegación. Había participado en la misión de reconocimiento a la que alude el título del manuscrito, y en el mismo desarrolla métodos e instrumentos para realizar una náutica entendida científicamente, e incluso utiliza una metodología próxima a la ciencia moderna, como al refutar la teoría de Figueiredo acerca de la relación lineal entre la longitud geográfica y la variación magnética de la aguja náutica. En este artículo se desarrollan algunas conclusiones a las que permite llegar un análisis del manuscrito. Se centra en la exposición que Diego Ramírez realizó en el mismo acerca de un procedimiento útil para los pilotos del cálculo de la latitud de un lugar por medio de alturas extrameridianas del Sol, lo cual implicaba un gran avance en los métodos de navegación de la época. También se esbozan las motivaciones e intereses geopolíticos de la monarquía hispana para promover la expedición de reconocimiento que hizo posible el manuscrito y las innovaciones que contiene.

PALABRAS CLAVE: Historia de la cartografía; Historia de la náutica; Ciencia española del s. XVI; Historia de la astronomía; Historia de las matemáticas.

CONTRIBUTIONS TO THE CALCULATION OF LATITUDE IN THE EARLY SEVENTEENTH CENTURY SPANISH NAVIGATION CONTAINED IN THE MANUSCRIPT OF DIEGO RAMÍREZ DE ARELLANO: "RECONOCIMIENTO DE LOS ESTRECHOS DE MAGALLANES Y SAN VICENTE, CON ALGUNAS COSAS CURIOSAS DE NAVEGACIÓN" (1621)

ABSTRACT: In 1620, was named chief pilot of the Casa de Contratación de Sevilla Diego Ramírez de Arellano, author of a manuscript entitled Reconocimiento de los estrechos de Magallanes y San Vicente, con algunas cosas curiosas de navegación. He had participated in the reconnaissance mission referred by the title of the manuscript, and in that book developed same methods and tools for a nautical understood scientifically, and even used a methodology close to modern science, as in refuting the theory about Figueiredo of the linear relationship between longitude and magnetic variation of the needle navigation. This article is based on some conclusions that can get an analysis of the manuscript. It focuses on the exhibition held at the Diego Ramirez same about a useful procedure for pilots of calculating the latitude of a place by the Sun extrameridianas heights, which meant a breakthrough in navigation methods of the time. It also outlines the motivations and geopolitical interests of the Spanish monarchy to promote scouting expedition that made the manuscript and the innovations it contains.

KEY WORDS: History of Cartography; Nautical history; Spanish Science in 16th Century; History of Astronomy; History of Mathematics.

Copyright: (c) 2013 CSIC. Este es un artículo de acceso abierto distribuido bajo los términos de la licencia Creative Commons Attribution-Non Commercial (by-nc) Spain 3.0. 
En un clásico libro de Martín Fernández de Navarrete (Fernández Navarrete, 1846) encontramos una amplia exposición de los logros conseguidos por Diego Ramírez de Arellano en el campo de la ciencia náutica en el manuscrito del que vamos a tratar ${ }^{1}$. En la obra de Pulido Rubio El Piloto Mayor (Pulido Rubio, 1950) se ofrece una semblanza de la vida de este personaje, y se centra en la glosa de los aspectos sociopolíticos que motivaron la expedición al estrecho de Magallanes en la que participó Diego Ramírez y que dio lugar posteriormente al manuscrito. Además de esas referencias que podemos llamar clásicas, la importancia de Diego Ramírez ha sido reclamada recientemente en textos como Tecnología de la navegación en la España renacentista (López Piñero, 2007); o en el artículo La expedición de los Hermanos Nodales y el cosmógrafo Diego Ramírez de Arellano (Vicente Maroto, 2001, p.p. 7-28). También es posible hallar más referencias puntuales a Diego Ramírez en otras obras, por ejemplo en Historia y leyenda de la aguja magnética (Martínez Hidalgo, 1946), que se refiere a la intervención de Diego Ramírez en cuanto al problema de la declinación magnética, o en la obra La Cartografía Náutica Española en los siglos XIV, XV y XVI (Cerezo Martínez, 1994) que alude al mapa del estrecho realizado a partir de los datos tomados en la expedición, o en otras obras en las cuales se le menciona a raíz de su nombramiento como Piloto Mayor de la Casa de Contratación de Sevilla (González, 1992). Últimamente ha sido editada una transcripción del manuscrito con un estudio preliminar que se centra en la biografía de Diego Ramírez (Soler Pascual, 2011), y se ha leído una tesis doctoral en la que se realiza un estudio del manuscrito centrado en los aspectos más técnicos de navegación y cartografía, así como una transcripción del mismo (Díaz Hernández, 2011).

\section{ESTRUCTURA DEL MANUSCRITO}

El manuscrito se divide en cuatro partes. En la primera parte, titulada Relación diaria de lo sucedido en el viaje, encontramos un diario de a bordo que incluye minuciosos datos astronómicos a partir de los cuales obtendrá coordenadas geográficas, además de datos sobre corrientes predominantes, datos sobre mareas, sobre vientos y sobre accidentes geográficos de la costa más meridional del continente americano tales como Cabos, bahías, islas, bajíos..., además de un derrotero de retorno a España. En la segunda parte, titulada De lo observado en el viaje Diego Ramírez realiza una ordenación de las observaciones empíricas recopiladas durante el viaje, de tal modo que las presenta en forma de tablas útiles para los navegantes que realicen el paso del estrecho de Magallanes. Por ejemplo, ordena todas las observaciones sobre mareas (horas de bajamar, pleamar, mareas vivas y sentido de la corriente de la marea según la zona del Estrecho) en una tabla en la que es posible averiguar para cualquier hora del día en una fecha del año el estado de la marea en cada una de las bocas del estrecho; y lo mismo hace con los valores deducidos sobre la variación magnética, estableciendo una tabla de declinaciones que permite hacer las correcciones de rumbo adecuadas durante la navegación del estrecho de Magallanes; así como también presenta una minuciosa tabla de coordenadas geográficas a partir de las cuales podrá elaborar la cartografía. Al tratarse de las primeras recopilaciones de este tipo de datos en esa zona del planeta, dichas tablas presentadas en esta segunda parte del manuscrito tienen una relevancia especial, pero sobre todo porque no se limita a presentar y ordenar datos, sino que en la tercera parte, titulada De la doctrina con que se hicieron las observaciones de este viaje, se dedica a justificar desde la teoría cómo se interpretan estas observaciones, y cómo se pueden elaborar procedimientos generales para obtener nuevas mediciones, o sobre cómo es posible desde el conocimiento de leyes generales (por ejemplo Teoremas de la Geometría) abordar problemas concretos de navegación ${ }^{2}$. El caso más destacable es el de la obtención de la altura del polo a partir de alturas extrameridianas del Sol, ya que expone un algoritmo útil (y que según el manuscrito se presenta por primera vez para utilidad de los navegantes ${ }^{3}$ ) deducido a partir de la trigonometría esférica más elaborada de su época. Es decir, logra encajar la resolución de un problema náutico (cálculo de las coordenadas del buque) dentro del marco general de la Geometría.

Destaca en este manuscrito y al mismo tiempo lo hace un texto muy diferente a los manuales que a lo largo del siglo XVI se habían destinado a la enseñanza de los pilotos (los de Pedro de Medina, Cortés, Zamorano...), el detallado análisis e interpretación desde la teoría de los datos obtenidos durante el viaje al estrecho de Magallanes, a partir de los cuales se elabora la carta náutica de la zona geográfica que se extiende desde el Río de la Plata hasta la Tierra del Fuego (entonces denominada Isla de Xàtiva) ${ }^{4}$. También destaca la detallada exposición de la infraestructura experimental utilizada, con la descripción de todos y cada uno de los instrumentos utilizados en el proceso de medición. Creemos que es suficiente todo lo comentado hasta aquí sobre el manuscrito para hacerlo merecedor de un extenso y minucioso análisis. Sobre todo porque se separa de otros textos sobre náutica en cuanto a la preocupación por encajar datos empíricos recopilados sobre el terreno y la teoría disponible para organizarlos e interpretarlos. Y ello fue posible porque Diego Ramírez encarna un nuevo tipo de Piloto Mayor: a diferencia de su antecedente, Zamorano, por ejemplo, Diego Ramírez cuenta con una sólida formación científica (no menor que la de Zamorano, como se demuestra en el manuscrito), pero además ha tenido amplia experiencia como navegante, algo de lo que adolecieron los pilotos mayores a partir de Alonso de Chaves, nombrado en $1552^{5}$. 
No hay que perder de vista que la posibilidad de que pudiera realizarse este tipo de manuscrito (que entremezclaba varias disciplinas) se basaba en última instancia en el interés de la corona española por controlar una zona geográfica ante las hostilidades de holandeses e ingleses en ese momento histórico. Es decir, este libro no es posible si previamente no existe una orden del monarca (en forma de Real cédula de 26 de agosto de 1618) para reconocer el estrecho de Magallanes, lo que a su vez significa que sin esta orden del monarca no es posible poner a prueba nuevas maneras de relacionar la teoría y la práctica en cuestiones de náutica, de cartografía y geografía, lo que a su vez significa que sin el interés del monarca no es posible adquirir nuevos datos que pongan en cuestión antiguas teorías que se ajustan a antiguos datos pero no a los nuevos.

Ello nos hace ver una vez más la relación casi inextricable entre ciencia e intereses del Monarca que atravesó todo el quehacer científico en la península durante el siglo XVI. Al hilo de esta cuestión, Navarro Brotons y Salavert Fabiani llaman la atención sobre una posible revisión historiográfica que otorgue el relieve que verdaderamente tuvo la actividad desarrollada en diferentes ámbitos del saber en la época previa a la Revolución científica, no solo en el sentido de atender al papel relevante que disciplinas como la geografía o la cartografía tuvieron como elementos desencadenantes de la misma, sino también por la vinculación que tuvieron estas mismas disciplinas con las necesidades administrativas de la Monarquía (Navarro Brotons y Savater Fabiani, 2006, pp. 209-228).

En la Casa de Contratación de Sevilla, desde su fundación real en 1503, y a lo largo del siglo XVI se experimenta un proceso en lo referido a la transmisión de enseñanzas náuticas, a los procedimientos requeridos para expedir las licencias de los pilotos que habrían de intervenir en la carrera de Indias, y a los criterios por los que se consideraba óptima la decisión de nombrar a los pilotos mayores. Un interesante trabajo (Sandman, 2001) muestra que en la primera mitad de siglo este proceso responde a una tensión entre dos polos o estilos de entender la náutica: el estilo tradicional, que consiste en navegar de modo empírico, sin atenerse a principios científicos a partir de los cuales resolver o afrontar situaciones concretas de navegación; y el estilo de los denominados por Sandman Theory-proponents, individuos con excelente formación en cosmografía y que vieron la posibilidad de aplicar con eficacia su conocimiento geográfico, astronómico y matemático a los problemas con que se enfrentaba la navegación trasatlántica, fundamentalmente en lo referido al cálculo de las coordenadas geográficas de la embarcación y la determinación de su rumbo para desplazarse al lugar deseado.
La deficiente navegación que ofrecía para estas rutas de Indias el estilo tradicional generaba numerosos naufragios y por tanto pérdidas cuantiosas no solo en vidas humanas sino también en mercancías a menudo de gran importe económico (Pérez-Mallaina Bueno, 1996). Este hecho, que afectaba a los intereses de la Corona, provocó una serie de medidas administrativas que no dejaron de sucederse a lo largo del siglo XVI, y que se tradujeron en la creación de una serie de instituciones que respondían en gran parte a la necesidad de mejorar los procedimientos de navegación.

En la segunda mitad del siglo, el Consejo de Indias potencia la figura de los cosmógrafos, a quienes considera útiles no solo para la náutica, sino también para realizar el padrón real, mapa en el que consten los lugares que pertenecen al monarca con su situación lo más exacta posible y especificada por sus coordenadas geográficas, y a partir del cual extraer las cartas náuticas que se utilizaban en las navegaciones transoceánicas. Ello dio pie a la ingente labor de Juan López de Velasco ${ }^{6}$, estudiada con detalle por M. Portuondo en lo referente a su significación política (Portuondo, 2005). Además, la Corona consideró de sumo interés crear la Academia de Matemáticas, con el fin, entre otras cosas, de perfeccionar los procedimientos e instrumentos náuticos (Vicente Maroto y Esteban Piñero, 1991).

En esta situación se llega al principio del siglo XVII, habiendo ocupado España a lo largo del XVI un papel preponderante en la elaboración de Regimientos Náuticos y otros textos destinados a la enseñanza de los pilotos (Medina, Cortés, Zamorano...). El Piloto Mayor de la Casa de Contratación de Sevilla, Zamorano, eminente representante del polo teórico, será reemplazado a su muerte por Diego Ramírez de Arellano, autor del manuscrito que es el objeto de este trabajo.

\section{CONTEXTO GEOPOLÍTICO QUE MOTIVA LA EXPE- DICIÓN EN QUE PARTICIPA DIEGO RAMÍREZ DE ARELLANO}

La Reforma protestante había desencadenado entre otras cosas el hecho de que Inglaterra se negara a aceptar la validez de las bulas de Alejandro VI que repartían los territorios descubiertos entre España y Portugal, excluyendo a otras naciones del reparto. Drake había atravesado el estrecho de Magallanes, incluso tomando posesión de la Tierra del Fuego en nombre de la reina Isabel, y el 15 de febrero de 1579 apareció en las cercanías del puerto del Callao logrando desembarcar a pesar de la defensa que se organizó contra él. Era evidente que si las flotillas de piratas comenzaban a dominar aquel paso, se encontrarían con un evidente peligro las colonias españolas situadas en el litoral del Pacífico. Por tanto, se trataba con aquella señal de hostilidad corsaria de comunicar a la corona 
española que no se aceptaban las reglas del juego que concedían la prioridad española y portuguesa sobre los territorios del Nuevo Mundo.

El ambiente de prevención que comenzó a rodear a la corona se muestra en una serie de documentos, de entre los cuales podemos tomar como especialmente significativo el conjunto de cartas del embajador Bernardino de Mendoza, en el cual se advierte continuamente al monarca de los movimientos hostiles de naves inglesas ${ }^{7}$. En el contenido de esta correspondencia se muestra una inquietud que habría de influir en las decisiones del monarca acerca de la geopolítica del estrecho de Magallanes, en cuyo decurso se inscribiría la expedición de los hermanos Nodal y Diego Ramírez con el encargo específico de realizar una cartografía del estrecho.

Pautas para la expedición contenidas en la Real Cédula de 28 de agosto de 1618

La pauta marcada a la navegación según la Real Cédula, en cuanto a derroteros a seguir durante la travesía una vez llegados a tierra americana es la siguiente:

1) Recorrer la costa de Brasil hasta el Río de la Plata.

2) Bajar desde el Río de la Plata costeando hasta el Cabo de las Vírgenes.

3) Embocar el estrecho de Magallanes hasta salir al Océano Pacífico (Mar del Sur) si las condiciones meteorológicas lo permiten, y reconocer la boca del estrecho de Magallanes por el extremo occidental, a 53으 de latitud sur, (latitud estimada según la Real Cédula).

4) Reconocer las islas que hay en esta boca del estrecho, y realizar un derrotero de la zona tomando señales de tierra a un lado y otro, sondando el fondo y «reconociendo si hay algún surgidero seguro en la costa».

5) Realizar la navegación del estrecho tomando alturas de polo y longitud en diferentes puntos del mismo.

6) Realizar un reconocimiento de la boca occidental del estrecho desde mar adentro, "para desde allí hacer el dicho reconocimiento y pintura de los aspectos y señales de la tierra y hecho esto, dando el tiempo lugar os volveréis por el mismo estrecho de Magallanes hasta salir a la Mar del Norte (Océano Atlántico), en busca del estrecho de Le Maire».

7) Si las condiciones meteorológicas lo permiten, avanzar hacia el sureste sin perder de vista la costa, "reconociendo y sondando los puertos y bahías que hallareis, poniéndolos en el derrotero con la señales que tuvieran las tierras y la altura de polo, longitud y variación de la aguja, siguiendo la costa hasta los 55 grados y dos tercios, que es donde se tiene aviso que está el estrecho de Le Maire».

8) Una vez avistado el citado estrecho, dice la Real Cédula que «entrando en el estrecho de Le Maire os llegareis a la costa que con más comodidad el tiempo os diere lugar reconociendo los puertos, bahías y surgideros, los fondos que tienen y si son limpios, poniendo cada cosa con mucha puntualidad en el derrotero».

9) A continuación se demanda el reconocimiento de la costa opuesta a la anterior del estrecho de Le Maire, hasta llegar a la boca oriental del estrecho de Magallanes, donde «con las mareas se ha de tener mucho cuidado en considerar lo que bajan y suben de aguas vivas y muertas, y a qué hora y día de Luna, poniéndolo en el libro del derrotero».

10) Asimismo se establece explícitamente en la Real Cédula cuál es la misión de Diego Ramírez: «En los puertos, bahías o cabos que fuere menester reconocer desde tierra se desembarcará Diego Ramírez con la guarda necesaria, poniendo las postas que convengan para descubrir los indios que vinieren a tierra, para que con más seguridad Diego Ramírez pueda sacar la línea meridiana, haciendo las observaciones necesarias y poniendo en los derroteros la latitud del polo, longitud y variación de la aguja que tuvieren las nuevas tierras que se descubrieren».

11) Con el plan de navegación detallado en los puntos anteriores el monarca demanda en la misma Real Cédula que «desde la boca del estrecho de Magallanes hasta el de Le Maire procuréis arribar a la costa y traer planta de toda ella y del mismo estrecho de Le Maire y su costa hasta la punta del Mar del Sur, que está en cincuenta grados, lo mejor que se pudiera». Esa demanda expresada en la Real Cédula convierte la expedición, en cierto sentido, en una navegación organizada científicamente con la que se persigue obtener la mejor cartografía de los estrechos de Magallanes y San Vicente (o Le Maire).

\section{SOBRE EL CÁLCULO DE LA ALTURA DEL POLO POR ALTURAS EXTRAMERIDIANAS DEL SOL}

Aunque en el manuscrito encontramos varios aspectos científicos de la náutica (entre los cuales destaca el estudio de la variación magnética, que lleva a Diego Ramírez a refutar con minuciosos resultados la teoría de Figueiredo sobre la relación lineal entre longitud geográfica y declinación magnética), seleccionamos en este artículo el análisis con cierto detalle 
Figura 1. Mapa de Diego Ramírez de Arellano de la zona reconocida durante la expedición

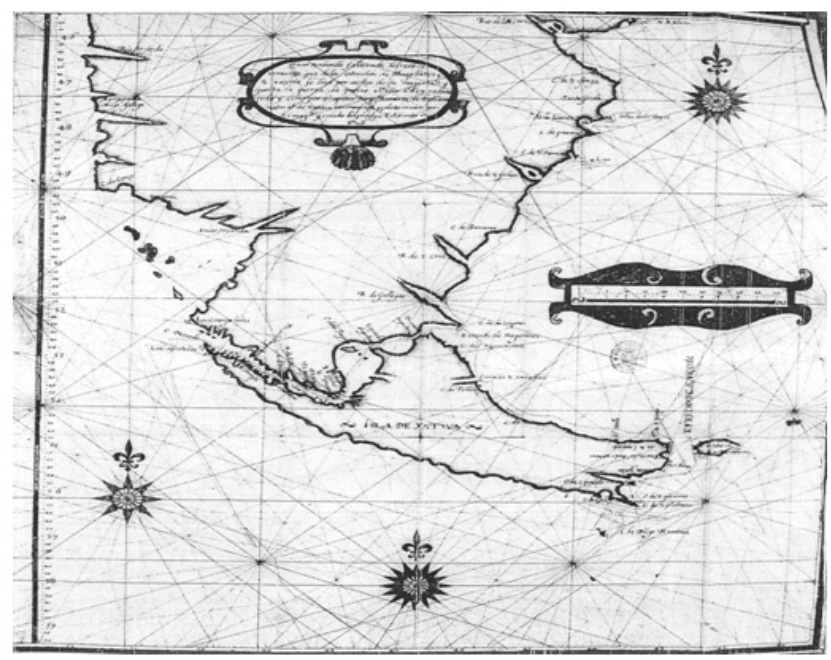

del cálculo de la latitud por alturas extrameridianas, ya que es en este apartado del manuscrito donde Diego Ramírez muestra el máximo dominio de las herramientas matemáticas conocidas en la época aplicadas a la resolución de problemas náuticos. Su conocimiento teórico de los fundamentos de la trigonometría esférica en los textos más elaborados del momento (Regiomontano, Gemma Frisius, Pedro Núñez, García Céspedes, Clavius...) le permite exponer todos los métodos conocidos hasta la fecha sobre el complejo problema del cálculo de la latitud del lugar por medio de alturas extrameridianas del Sol, y no solo eso, sino fundamentar las objeciones que se les puede achacar para su uso práctico en el mar durante las navegaciones. En este sentido vuelve a mostrar su capacidad de teorizar con una visión pragmática, superando así los límites de esos procedimientos de "salón", que aunque impecables matemáticamente adolecen de una falta de implementación en la práctica náutica; y al mismo tiempo superando también las limitaciones teóricas que restringían a los pilotos el acceso a una navegación científica, basada en un corpus bien trabado, del cual se puede deducir la resolución concreta de un problema encajándolo en un marco general y abstracto. Es la muestra de esa síntesis de la que habla Sandman y que hemos ido comentando a lo largo de este texto.

Sobre el problema en concreto que aborda Diego Ramírez en este apartado del manuscrito, la motivación del mismo es expresada en estas líneas que reproducimos: "Después que la aguja de demarcar dio claridad a la investigación, y mediante ella y los demás instrumentos de esta arte se han emprendido golfos tan grandes, y descubierto tantas y tan remotas naciones, por cuyo tratado y conocimiento se ven los mares arados de continuas navegaciones, se ha fomentado el asunto de este capítulo de tal suerte que viendo los matemáticos la utilidad que se seguiría si se supiese en la mar la altura del polo a cualquier hora el día han procurado buscar diversos modos para ello, ya inventando instrumentos, ya dando doctrina de los inventados, ya por números, ya con sola regla y compás, intentando cuantos caminos les ha sido posible para poder dar reglas a los marineros mediante las cuales supieran la altura del polo a cualquier hora del día como les dieron los que hoy usan.

En la mar, el mejor y más fácil modo que hasta hoy se ha conocido de saber la altura del polo es al punto del mediodía; con todo, o porque este no se sabe con la puntualidad que es justo, o porque algunos gustaran de no estar atenidos a él, o por lo que algunas veces sucede el no haber Sol al punto del mediodía, después de haber pasado algunos días de tormenta, y ser de importancia la altura del polo para saber con más seguridad dónde se hallan, o por llegar a algunos cabos y puertos después de mediodía, e importando saber su altura no poder detener en él, o deteniéndose estar el cielo turbado al mediodía, será bueno, ya que no para los pilotos que solo se contentan con sus reglas ordinarias, para los que fueren más curiosos poner algunos modos con que esto se sepa en la mar» (folio 136).

Como vemos, la restricción en cuanto al conocimiento de la latitud del lugar que supone el procedimiento de la observación de la altura del Sol a su paso por el meridiano motiva por sí misma el desarrollo de procedimientos basados en la observación de alturas extrameridianas. Ello implica pasar de una situación geométrica que no exige el análisis de triángulos esféricos (latitud a partir de la altura meridiana del Sol), a otra en la cual sí es necesario resolver triángulos esféricos (latitud a partir de alturas extrameridianas del Sol). 
En el siglo XVI comienzan a aparecer en tratados de matemáticas, de astronomía y de náutica procedimientos para calcular la latitud a partir de alturas extrameridianas. Por ejemplo, Pedro Nunes, en su Tratado da Sphera (Coimbra, 1537) ofrece un primer intento de facilitar este cálculo a los navegantes por medio de un instrumento, llamado poma o esfera, que más adelante detallaremos. A principios del siglo $\mathrm{XVI}$ ya era este un método conocido y familiar para los astrónomos, pero el procedimiento expuesto por $\mathrm{Nu}$ nes es original y dio paso a una sucesión de procedimientos aplicables en la navegación, entre los cuales se encuentra el de Diego Ramírez que posteriormente expondremos. Antes de centrarnos en el método de Nunes, comentemos previamente los citados por Diego Ramírez antes de llegar a este matemático: Apiano, Juan de Rojas, Figueiredo y Frisius.

Sobre el de Pedro Apiano, escribe Diego Ramírez: «en la primera parte, proposición 3 de su Cosmografía, dice que tomando la altura del Sol a una hora conocida se sabrá la altura del polo». Este procedimiento, en el cual es necesario conocer la altura del Sol y la hora en la cual se ha determinado esta altura, además de la declinación, es desechado por Diego Ramírez, ya que al no ser posible determinar con exactitud la hora, a causa de la pobre tecnología relojera de la época, al aplicar este procedimiento al cálculo de la altura del polo se obtendría un error considerable, estimando Diego Ramírez errores de más de 50, lo cual significa que «por mala que sea la fantasía no se errará tanto ${ }^{8}$. Es decir, que con los errores cometidos para fijar las coordenadas geográficas por medio del rumbo y la distancia estimados por el piloto, se llegaría a mejores resultados que con este método que comenta Apiano para calcular la altura del polo con una altura extrameridiana del Sol.

Continúa Diego Ramírez con el procedimiento de Juan de Rojas, expuesto en el libro 2, capítulo 27 del Astrolabio, donde «enseña a sacar la altura del polo por el conocimiento de la hora en que el Sol sale o se pone en la parte del mundo donde se hallaran, y en el capítulo 46 por la ascensión oblicua de cualquier signo ${ }^{9}$. Es evidente que si Diego Ramírez deshecha el método anterior por la inexactitud en la determinación de la hora por los relojes de la época, por una razón similar desecha este procedimiento de Juan de Rojas, ya que "los cuales modos tiene los mismos inconvenientes que el pasado sino mayores, pues ni la hora en que el Sol sale o se pone, ni la ascensión oblicua de cualquier signo se puede saber sin conocer primero la altura del polo» ${ }^{10}$.

A continuación trata el manuscrito del procedimiento expuesto por Figueiredo en su Arte de Navegar, que se basa en la determinación de la altura del polo conociendo la amplitud del Sol, o separación angular en el orto o en el ocaso del Este u Oeste verdadero, lo cual fue tratado por Diego Ramírez en el capítulo anterior. Pero el cálculo de la latitud por medio de la amplitud, como asegura Diego Ramírez, tiene dos inconvenientes básicos, que implican graves errores en el resultado final de la latitud: 1) la dificultad de fijar la meridiana que proporcione la línea Este-Oeste del lugar respecto a la cual medir esa amplitud, a causa del desconocimiento de la variación magnética del lugar, y 2) debido a que cuando la declinación solar es menor de 17으, navegando entre los trópicos, estima Diego Ramírez, el error en la amplitud es más probable por que este ángulo va disminuyendo, llegando a ser muy apreciable. Textualmente dice nuestro autor: «Figueiredo, en el final del capítulo 8 de su Arte de Navegar enseña a saber la altura del polo por la amplitud del Sol, y las tablas que están puestas en el capítulo precedente, la cual enseña a sacar por la aguja de demarcar, y la variación, cuya doctrina no quiero detenerme a refutar de propósito, solo diré que se le echa bien de ver a este autor no haberlo experimentado en la mar, ni ponderado en tierra, pues se atrevió a enseñar cosa que por ella se puede errar la altura en más de $30 \mathrm{gr}$ errando la amplitud en solo un grado, cosa muy posible; y aunque tuviera la aguja fija con la cual se supiera con más precisión la amplitud del Sol no es practicable en la mar, pues navegando uno entre los trópicos en tiempo que el Sol tuviese menos que 17gr de declinación, errando la amplitud cuando marcase el Sol por la aguja, en 20 minutos, cosa no solo posible sino muy cierta en la mar, podía venir a errar la altura en 10gr porque si teniendo el Sol 17gr de declinación, se hallase que tenía la misma amplitud habiendo de tener $17 \mathrm{gr} 20 \mathrm{~m}$, por su cuenta se hallaría en la línea, o en un grado de altura, habiendo de hallarse en 11gr, que son los que competen a $17 \mathrm{gr} 20 \mathrm{~m}$ de amplitud, y es tanta la verdad esta, que teniendo el Sol 6gr de declinación, con solo 20 minutos de yerro al marcar el Sol, será posible errar la altura en más de $18 \mathrm{gr}$, cosa exorbitante e indigna de hombre de esta profesión» (fol. 137).

Por tanto, a partir de las estimaciones de Diego Ramírez, que arremete una vez más contra los métodos del portugués Figueiredo, este modo de cálculo de la latitud es desechable por el análisis de error efectuado en el párrafo anterior. Pero además, Diego Ramírez tiene en cuenta la falacia de la teoría de Figueiredo sobre la variación magnética, lo que sería una fuente añadida de error en la determinación de la latitud, pues llevaría a la determinación errónea de las meridianas (cuya correcta determinación exige conocer con exactitud la variación magnética el lugar). Escribe Diego Ramírez: "Mas para saber esta amplitud, o se ha de valer de las variaciones observadas por los pilotos portugueses, o las ha de observar de nuevo: $\mathrm{Si}$ por las observadas se ha de cometer grandísimo yerro, porque todas están a grados enteros, dándonos a 
entender en esto que no hacían caso de los minutos, y así por lo menos han de estar erradas unas de más y otras de menos de $30 \mathrm{~m}$; si estas variaciones las han de observar de nuevo, o será con una o con dos observaciones; si con una no pueden saber la variación que no sepan primero la altura del polo, si con dos será por la mañana la una, no pudiéndose hacer la otra por el mediodía (en cuyo tiempo se puede observar la altura), pues ha de ser a la tarde, y siendo así no será cordura dejar lo fácil por lo dificultoso, lo cierto por lo incierto» (fol. 137).

Sobre el método expuesto por Frisius en el capítulo 42 del Astrolabio Católico, dice Diego Ramírez: «...enseña a saber la altura del polo con sola una observación a cualquier hora del día, presuponiendo conocida la línea meridiana, y como esta no se puede conocer sino por una aguja fija (la cual aunque muchos la han ofrecido ninguno la ha dado) y ésta no la tenemos, así no será practicable en la mar este modo, de donde se colige que entre los provechos que la aguja fija daría a la navegación no sería el menor poderse saber por él la altura del polo a cualquier hora del día con sola una observación» (fol. 137).

A partir de aquí, Diego Ramírez asume el hecho de que sea cual sea el método para calcular la latitud del lugar por alturas extrameridianas del Sol, se debe eliminar la fuente de error que supone la determinación de la meridiana por ser incierta o desconocida la variación magnética del lugar, y que un modo de lograrlo es tomar dos alturas extrameridianas, ambas antes o después del mediodía, y el ángulo que forman los verticales de la posición del Sol cuando se toman ambas alturas. En efecto, de este modo, como vamos a ver al tratar el caso de Pedro Nunes, se logra eliminar esta dependencia de una magnitud que o no se conoce o es incómodo determinar. Dice Diego Ramírez: "Finalmente, vistos los inconvenientes de los modos pasados, se vienen a resolver todos que faltando aguja que nos dé la meridiana cierta, no se puede saber la altura del polo a cualquier hora del día si no es por dos observaciones» (fol. 138).

Sobre el método de Pedro Nunes comenta que «no es practicable». Veamos en qué consiste este método. Consiste en construir una esfera con punzones en dos extremos de un diámetro, y que servirán de eje de giro a un semicírculo fabricado en latón y de radio un poco mayor que el de la esfera. Este semicírculo debe incluir una doble graduación de 0 a 90 grados (hacia el Norte y hacia el Sur), estando el cero de la escala en el medio del semicírculo. En la superficie de la esfera se representa también un semicírculo máximo que pase por los punzones, que representará el meridiano del lugar y que estará asimismo graduado. Por último se representa un círculo máximo perpendicular al eje de giro que representará el horizonte del lugar.
Con este instrumento ideado por Nunes se puede resolver gráficamente el problema de la latitud por medio de una altura extrameridiana del Sol. Se debe conocer para utilizar este método:

1) Por observación con astrolabio o cuadrante la altura del Sol.

2) Por medio de unas tablas la declinación solar de la fecha.

3) El ángulo acimutal del Sol ${ }^{11}$, que es posible medir con un teodolito y una brújula, siempre que se pueda conocer con exactitud la variación magnética del lugar.

Con estos tres datos, y teniendo en cuenta la figura 2 que aparece en la página siguiente, se procede como sigue:

a) Sobre el círculo del horizonte, a partir del cero de la escala se traza un arco BA que sea igual al acimut medido.

b) En el semicírculo se toma el arco AS, que debe ser igual a la altura medida del Sol sobre el horizonte.

c) Con centro en S y con un radio SC, que debe ser igual a la distancia al polo del Sol, es decir: 90응 de declinación, se traza con un compás esférico el círculo menor CC', que estará formado por el conjunto de puntos donde puede estar el polo, ya que todos esos puntos cumplen que tienen la misma distancia polar al Sol. Es decir, esa circunferencia es el lugar geométrico del Polo.

d) Como al mismo tiempo el polo celeste pasa por el meridiano del lugar, obtenemos dos puntos de intersección, $C$ y $C^{\prime}$ que pueden corresponder a la solución de nuestro problema: o bien la latitud es $B C$ o $B C^{\prime}$, como se observa en la figura 2 .

e) Para decidir entre las dos posibilidades, se recurre a la estima de la posición del lugar, es decir, el piloto lleva en su cuaderno de bitácora una estimación diaria de los recorridos de la nave por rumbo y velocidad. Ello le hace deducir un cierto valor de la latitud: el más próximo será o $\mathrm{BC}$ o $\mathrm{BC}^{\prime}$, lo cual permite elegir un valor usando este procedimiento.

Utilizando el concepto de triángulo de posición podemos explicar el fundamento basado en trigonometría esférica de este método. Supongamos la siguiente esfera, en la que aparece el triangulo de posición formado por el Sol, el zenit del lugar Z y el polo celeste P. Lo que hace el método de Nunes es formar este triángulo de posición, en el cual podemos determinar los lados 90 - h (90 - altura solar) y 90 - d (90 - declinación) y el ángulo 180 - A (180ㅇ - azimut solar). Tengamos en cuenta que el azimut astronómico se define 
Figura 2. Esfera o poma de Pedro Nunes

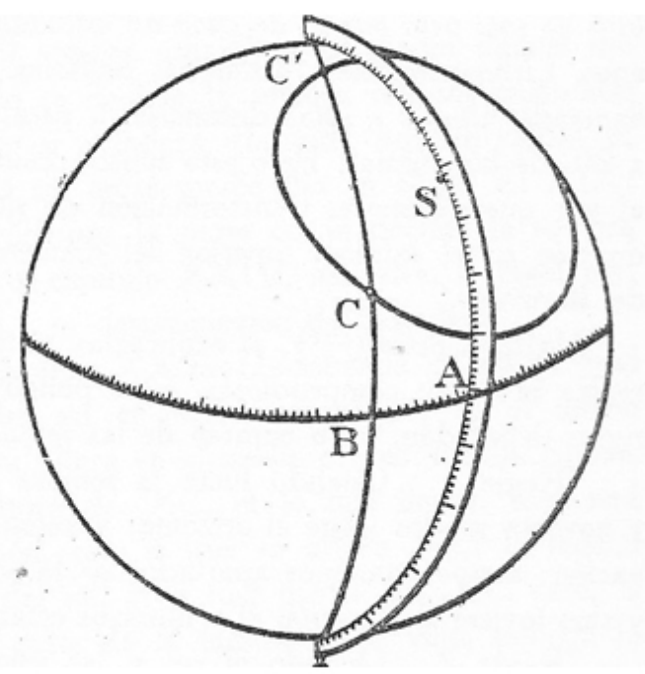

como el ángulo medido sobre el horizonte del lugar que forma el Sur del lugar y el punto de intersección del vertical del astro con el horizonte.

Este ángulo es el que corresponde al arco $A B$ de la figura 2, y también corresponde al ángulo en el zenit $Z$ de la figura 3. De este modo lo que hace el método de Nunes es reproducir a escala el triángulo de posición que sobre la esfera celeste determina en ese momento el Sol, y que vemos en la figura 3:
A pesar de que este método gráfico es muy práctico, y supone una innovación respecto de lo que a un piloto de la época parecerían farragosos e ininteligibles procedimientos incluidos en los libros de los astrónomos, Diego Ramírez asegura sumariamente que este procedimiento no es practicable, y aunque no explica las razones, lo afirma seguramente por la inexactitud en la media de la latitud del lugar derivada de los siguientes factores: a) el tamaño reducido del globo, b) las diferentes divisiones en las graduaciones debido a

Figura 3. Triángulo de posición del Sol

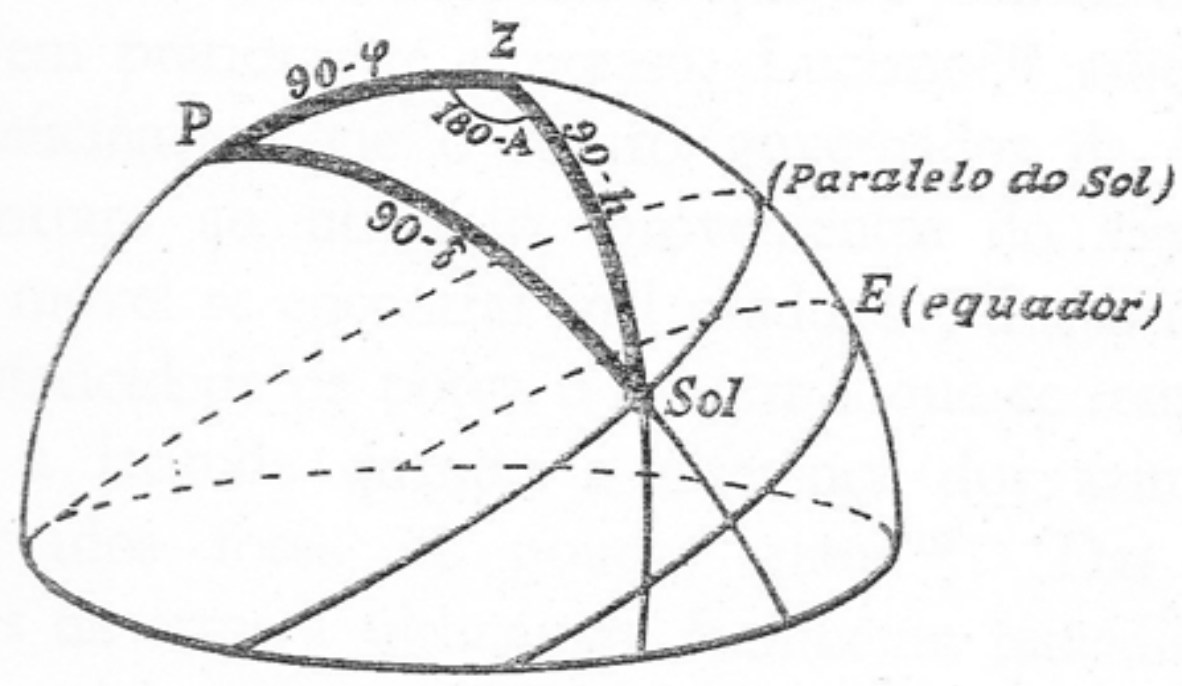


la inherente imposibilidad de lograr divisiones iguales, c) la dificultad de determinar con exactitud el acimut solar en la nave, entre otras cosas por el frecuente desconocimiento de la declinación magnética.

El mismo Pedro Nunes ya tuvo en cuenta estas limitaciones y trató de solventar la tercera de ellas tomando el azimut solar en dos posiciones diferentes del astro. Está documentado el hecho de que este método se utilizó en la mar en 1538 por Juan de Castro ${ }^{12}$.

Diego Ramírez expone a continuación el método elaborado por García de Céspedes en su Regimiento de Navegación, capítulo 8 , titulado "Donde se enseña la fábrica de un instrumento con el cual se sabrá la altura del polo a cualquier hora, y otras operaciones». Método que también considera impracticable por estar sujeto a graves errores a causa de la «falacia del papel». Veamos en qué consiste este método. Se trata de representar en dos círculos de papel una trama plana de meridianos y paralelos por medio de una proyección estereográfica ${ }^{13}$ : uno de ellos, el $A B C D$ representa el horizonte del lugar, el zenit y el nadir; los verticales o círculos máximos que pasan por el polo y el zenit, y los círculos menores paralelos al horizonte o almicantarates; y el segundo círculo abcd contiene el ecuador celeste, meridianos y paralelos celestes, y los polos celestes.

Con esta representación gráfica, como veremos, se debe señalar el Sol en el círculo ABCD con sus coordenadas horizontales: altura del Sol y azimut, que se han medido sobre el lugar del que se quiere conocer la altura del polo (se toman dos alturas y dos azimuts); y se superpone este círculo con esas señales al círculo abcd, con la condición de que las señales se superpongan al círculo de declinación que tiene el Sol en la fecha y que se toma en el círculo abcd. En esas condiciones es fácil comprobar geométricamente que el punto $A$ indica la altura del polo o latitud del lugar. En realidad se trata de un procedimiento plano análogo al de Nunes en tres dimensiones. EI aspecto de este instrumento es el de un planisferio obtenido por proyección estereográfica, como vemos en la figura 4.

Figura 4. Instrumento expuesto en Regimiento de Navegación, de García de Céspedes

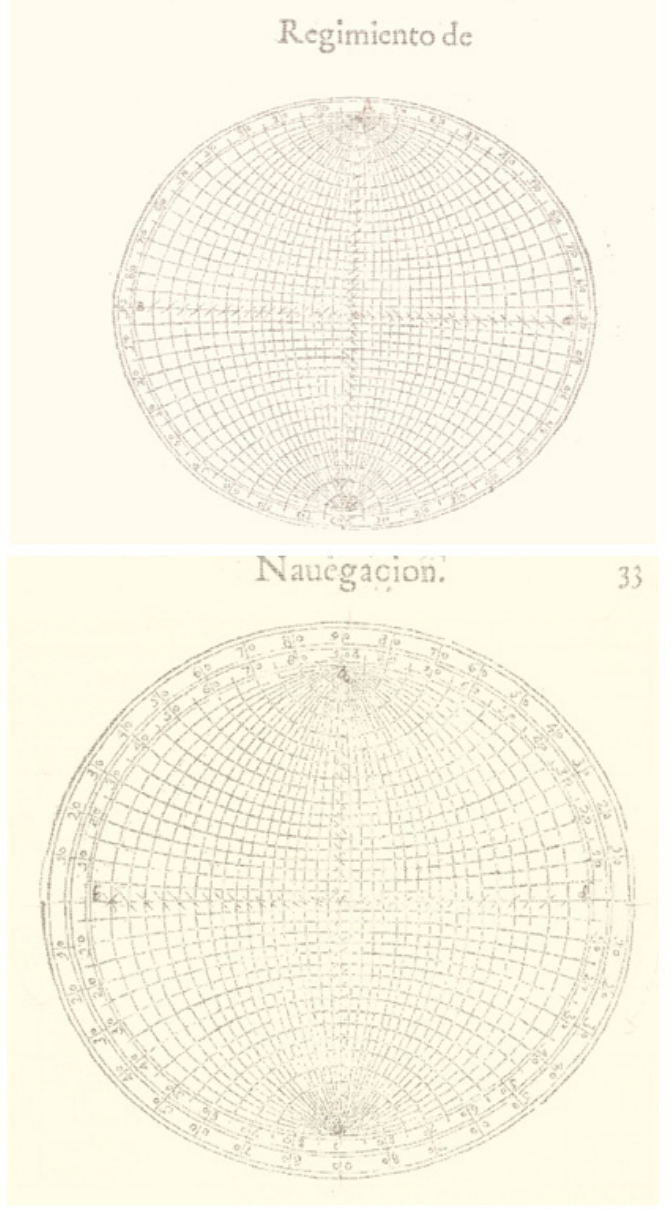


Este es el instrumento y el método de uso ideado por García de Céspedes, que puede superar apenas en precisión al método propuesto por Nunes, pero que en definitiva se trata de un método gráfico que no acepta Diego Ramírez como idóneo, ya que «está sujeto a algunos errores por la falacia del papel». Si lo que persigue Diego Ramírez es una precisión de grados y minutos, como asegura más adelante, es cierto que estos dos métodos que representan a escala (bien en una esfera o bien en círculos) los elementos del triángulo de posición Sol-Zenit-Polo Celeste, implican una inevitable inexactitud a causa del material mismo del instrumento: el globo no será perfectamente esférico ni las divisiones de las graduaciones exactamente iguales, o bien en los planisferios de Céspedes la superposición de los dos planisferios y los giros entre ellos inevitablemente conducirán a errores de hasta un grado. Es decir, son errores instrumentales, pero Diego Ramírez persigue un algoritmo que permita dar la latitud con precisión de minutos. Tengamos en cuenta que un objetivo fundamental de la expedición es la cartografía del estrecho de Magallanes, y para lograr una cartografía óptima de la zona necesita coordenadas geográficas lo más exactas posibles. Es decir, no solo busca un procedimiento que proporcione al navegante un modo cómodo de determinar la latitud, sino que también está buscando sentar un procedimiento que ofrezca la exactitud que requiere la confección de un mapa. En realidad, vemos a lo largo del manuscrito una preocupación por la exactitud del cartógrafo y la practicidad del piloto, rasgo que en este capítulo llega al máximo cuando nos ofrece una tabla de aplicación del cálculo de la latitud a Río de Janeiro, hallada con el procedimiento que ha ideado, obteniendo una latitud de 22038' que difiere mínimamente de la calculada actualmente $22054^{\prime 14}$.

Por esa motivación cartográfica, continúa exponiendo el método de Chistopher Clavius, que basado en una construcción gráfica con regla y compás, y usando resultados de trigonometría esférica, no se apoya en un instrumento como en los casos de Nunes y Céspedes $^{15}$. Este procedimiento toma como datos, igual que en los anteriores, dos alturas extrameridianas del Sol y la diferencia de azimuts entre las dos posiciones del Sol. Realiza la exposición del método por medio de un caso practico, realizado el 17 de enero de 1619 en el cabo de las Vírgenes, por la tarde, habiendo tomado alturas de 54ㅇ y 44으, con una diferencia de azimuts de 270 . Se basa en la construcción geométrica que hay en la figura 5 incluida en la página siguiente, tomada del manuscrito de Diego Ramírez. Sobre ella, los pasos que expone acerca del método de Clavius son: «Esto supuesto en un pliego de papel de la marca mayor se haga el círculo AFBE, de cualquier grandeza, advirtiendo que cuanto mayor fuere el círculo tanto más precisa saldrá la altura del polo que se busca; y tírese la línea HB, la cual representa la sombra de la menor altura del Sol, y tómese el arco AC de 44gr que el Sol tuvo de altura cuando formó la sombra HB. Del punto $C$ se cuenten los arcos CE, CD de 69gr20m, complemento de la declinación que el Sol tuvo aquel día (nótese que si la operación se hace en la parte del mundo contraria a la parte donde el Sol se halla como si estando a la banda del Norte se hiciese la operación en la parte del mundo del Sur la declinación que el Sol tuviere aquel día se añadirá a 90gr y todo junto será lo que el Sol está apartado del polo aparente). Sáquese el punto F, el cual será nadir de la sombra HB, y ha de distar una cuarta de cualquiera de los puntos $A$ y $B$; y del punto $F$, por los puntos $E$ y $D$ se tiren los rayos FH, FL, y del punto G, mitad de la línea HL, se haga el círculo dLv. Ídem tómese el arco AK, de $27 \mathrm{gr}$, que es el ángulo comprendido entre las dos sombras, y tírese la línea KM, que representa la sombra de la mayor altura, y tómese el arco KO, de 54gr, que el Sol tuvo de altura, y del punto $\mathrm{O}$, se cuenten los arcos OZ, OR, de $69 \mathrm{~g} 20 \mathrm{~m}$, distancia del Sol al Polo, y sacando el nadir N, que diste una cuarta de los puntos $\mathrm{KM}$, se tirarán NRP, $\mathrm{NiZ}$, y del punto T, mitad de la línea $\mathrm{Pi}$, se describirá el círculo eiv, el cual cortará al primer círculo en el punto $V$. Si del punto $X$, centro del círculo grande se tira la línea $\mathrm{mVs}$, será la meridiana; a la cual si se le saca su nadir, a, que diste una cuarta de los puntos, $m, s$, del cual tirada la línea av, corta en el círculo en el punto $\mathrm{t}$, y el arco st, que se hallará que vale $52 \mathrm{gr} 20 \mathrm{~m}$ largos es la altura que da este modo el cabo de las Vírgenes» (fol.139-140).

En este procedimiento la idea principal como vemos es la determinación de la meridiana (línea mvs en la figura 5) a partir de los datos tomados: dos alturas extrameridianas y la diferencia de azimuts. De ese modo se llega a calcular la altura del polo en la misma figura, que corresponde al arco st. La latitud encontrada para el cabo de las Vírgenes, que según Diego Ramírez son 5220' largos (interpretamos por largos que al medir con un instrumento análogo a un transportador de ángulos, la medida estaría más cerca de 5230' que de $522^{\circ} 0^{\prime}$ ), se desvía más respecto del valor correcto (5202') que el deducido para Río de Janeiro usando el procedimiento ideado por Diego Ramírez, y que a continuación expondremos. El problema de la exactitud está en que la representación gráfica, aunque sea del tipo desarrollado por Clavius, siempre está expuesta a errores a causa de los desvíos que pueden introducir la posición de la regla o el compás. Lo que va a establecer Diego Ramírez para superar esto, será un procedimiento numérico basado en un esquema al que se aplican resultados de trigonometría esférica. Lo importante es que el valor final de la altura del polo será deducido numéricamente, lo que logra eliminar los errores imputables al instrumento (esfera de Nunes o planisferios de García de Céspedes) o a la construcción geométrica de Clavius. 
Figura 5. Esquema usado por Diego Ramírez en su manuscrito

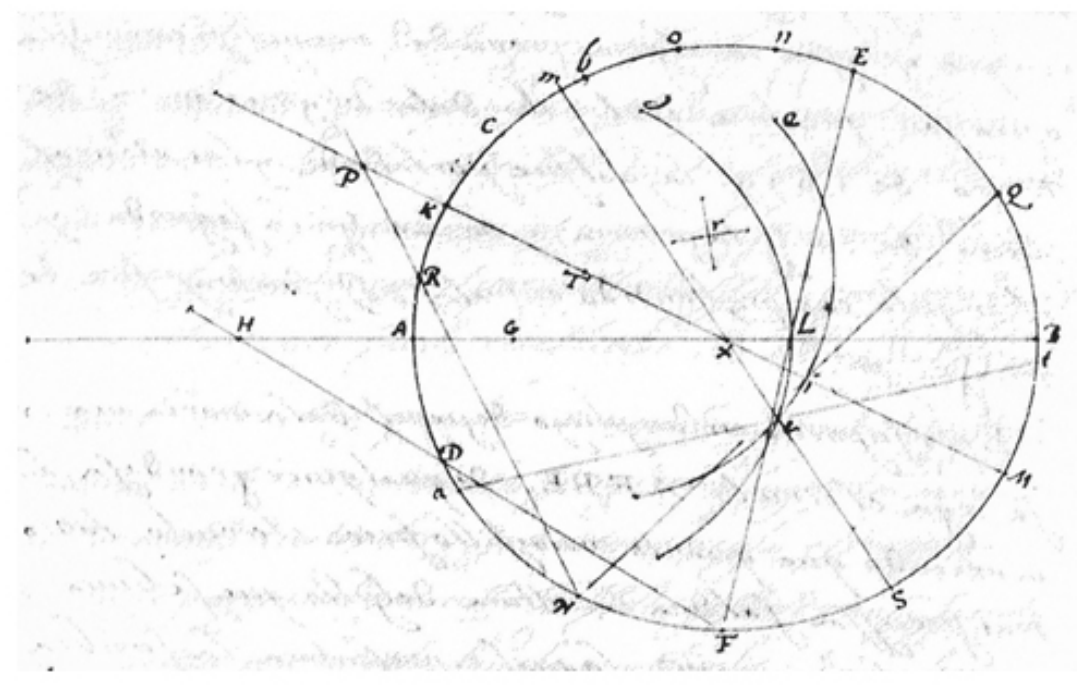

Dice Diego Ramírez: «Considerando que los dos modos anteriores no pueden dar entera precisión en los minutos, y que sería bueno dar doctrina que precediendo la observación cierta diese la altura del polo en grados y minutos exactos, no tanto por la mar en donde no importa tan rigurosa altura, sino para algunos Cabos y puertos en donde llegando después del mediodía, o faltando el Sol en los días que allí estuviesen se pudiese saber con precisión aquella altura, di en un modo que aunque laborioso es de provecho pues da rigurosa verdad, y como se ha de ofrecer cada día me pareció escribirlo para los escrupulosos que en algunas ocasiones les será de importancia, y harto más trabajoso sería no saber la altura del polo con certeza por faltar el Sol al mediodía y no tener doctrina con que saberlo, y solo servirá este modo a los que están ejercitados en contar y tienen cognición de las tablas de senos, tangentes y secantes» (fol.141).

Es de destacar esta reivindicación contenida en la última frase acerca de la ejercitación en el contar y en el manejo de tablas de senos, tangentes y secantes. Sabemos que los procedimientos numéricos y de cálculo en los que aparecen multiplicaciones y divisiones de razones trigonométricas pueden llevar a operaciones engorrosas por la cantidad de cifras decimales que pueden llegar a incluir dichas operaciones. Cualquier manual de historia de las matemáticas nos advierte del hecho de que esta necesidad de efectuar con cierta agilidad multiplicaciones y divisiones de números propició el desarrollo, hacia fines del siglo XVI, de los logaritmos. De hecho, John Napier comenzó alrededor de 1564 las investigaciones que le llevaron a la definición de logaritmo y a aplicar las propiedades de los mismos al cálculo.

El hecho de que los métodos analizados por Diego Ramírez prescindan de recurrir al cálculo numérico es indicio de la preferencia que había por las resoluciones gráficas y las construcciones geométricas, entre otras razones para evitar los engorros del cálculo. También, es obvio, por la herencia euclidea en el estilo de hacer matemáticas, que sabemos que se caracteriza entre otras cosas por el hecho de sobredimensionar la demostración basada en construcciones geométricas ${ }^{16}$. En este sentido debemos subrayar la no alineación de Diego Ramírez con los métodos clásicos si ello significa una ganancia en cuanto a exactitud en la derivación del valor de la magnitud que se busca, en este caso la altura del polo. Y en este capítulo lo demuestra con claridad, y ello nos muestra la capacidad de este autor para no encontrarse supeditado a la herencia recibida, y la predisposición a romper con ella a partir de correctas y detalladas argumentaciones. En ello hay que ver ese talante moderno que tanto iba a costar alcanzar en la ciencia española, pero que a través de textos como el que nos ocupa vemos que empieza a despertar.

El método que Diego Ramírez afirma haber dado con él, y que ofrecerá una precisión de minutos en el cálculo de la latitud incluye una figura con el único propósito de guiar visualmente el cálculo (no como una reproducción a escala en la cual se medirá la altura del polo como en los métodos de Nunes, Céspedes o Clavius), pero el resultado final se obtiene numéricamente, como efectuará en la práctica de aplicación del mismo al cálculo de la latitud de Río de Janeiro.

En la deducción aparecen tres triángulos esféricos, en cada uno de los cuales, a partir de los datos se va obteniendo otro lado o ángulo que será necesario para pasar al siguiente triángulo esférico, hasta llegar al triángulo que permite deducir la altura del polo ${ }^{17}$. Insistimos, primero hace una deducción geométrica, y posteriormente una aplicación numérica como vamos a ver. 
En sus propias palabras: «El asunto de él (del procedimiento de obtención de la altura del polo) es: Dadas dos alturas, ambas antes o después de mediodía, y el ángulo comprendido entre los verticales de las alturas, conocer la altura del polo. Llegando a tierra se nivelará el tablón del capítulo 10, y se pondrá de la suerte que allí se dice, y tomando la altura del Sol a grados enteros en el mismo punto se pondrá el torcal encima de la sombra, y se notarán los grados y minutos que corta en la circunferencia de aquel semicírculo, de allí a una o dos horas se volverá a hacer lo mismo, con lo cual se tendrán dos alturas del Sol, y el ángulo comprendido entre los dos verticales de las alturas que serán los grados que hubo entre las dos veces que se puso el torcal encima la sombra. Sea la mitad del meridiano superior $A B D$, y la mitad del horizonte $A D, y$ su polo sea B. La cuarta parte de la equinoccial EF y el polo del mundo aparente c. (Figura 6).

Los lugares del Sol al tiempo de las observaciones $\mathrm{HG}$, los verticales que pasan por ellos $\mathrm{Bi}, \mathrm{BR}$, las alturas del Sol, iH, RG, el ángulo comprendido entre los verticales de las alturas, GBH. Del polo del mundo, C, se tiren por los mismos lugares del Sol los círculos de declinación CG, CH, y por la 20. del 1. de Theodosio se tire el arco mayor GH. Digo que siendo conocidas las alturas del Sol iH, RG, y el ángulo Rbi, se conocerá la altura del polo DC, de este modo ${ }^{18}$ :

1) En el triángulo, $B G H$, están conocidos los dos lados BG, BH, complementos de las alturas del Sol, y el ángulo B comprendido entre los verticales de la observación, luego por la 64 de los Esféricos de Clavius conocido será el lado GH, y el ángulo, $\mathrm{BHG}^{19}$.
2) Ídem en el triángulo CGH, están conocidos sus tres lados, porque $\mathrm{GH}$ le acabamos de conocer, y $\mathrm{CH}, \mathrm{CG}$, son iguales por ser complementos de la declinación que el Sol tuvo el día de la observación, luego por la 63 de los Esféricos de Clavius se conocerá el ángulo $\mathrm{CHG}$, del cual quitamos el ángulo $\mathrm{BHG}$, conocido por el número primero, quedará conocido el ángulo $\mathrm{BHC}^{20}$.

3) Ídem en el triángulo $\mathrm{CBH}$, están conocidos los lados, HB, complemento menor de la altura, y HC, complemento de la declinación, esta conocida por el 2, el ángulo BHC; luego por la 64 de los Esféricos de Clavius, no se ignorará el lado BC, complemento de la altura del polo y por el consiguiente el arco $C D^{21}$, altura del polo que se busca» (fol.142).

Destaca en la exposición anterior de Diego Ramírez el hecho de que sea capaz de hacer un relato asequible de resultados de los libros de Clavius, es decir, que cualquier piloto con un poco de base matemática podría seguir el procedimiento que en el texto de Clavius sería inaccesible no solo por estar escrito en latín, sino porque técnicamente sus deducciones son complejas para el bagaje de un piloto de principios del siglo XVII (los textos al uso, como el de Zamorano, no llegan a tratar estos temas, y el más avanzado de Céspedes no desciende a casos concretos, como hará Diego Ramírez). A continuación el autor complementa la exposición con un algoritmo práctico de cálculo, que luego aplica al caso concreto del cálculo de la latitud de Río de Janeiro que realizó in situ consiguiendo una exactitud sorprendente para la época. Sin duda se trata de uno de los logros más reseñables de la náutica española de principios del siglo XVII.

Figura 6. Esquema usado por Diego Ramírez para aplicar su algoritmo de cálculo de la latitud por alturas extrameridianas

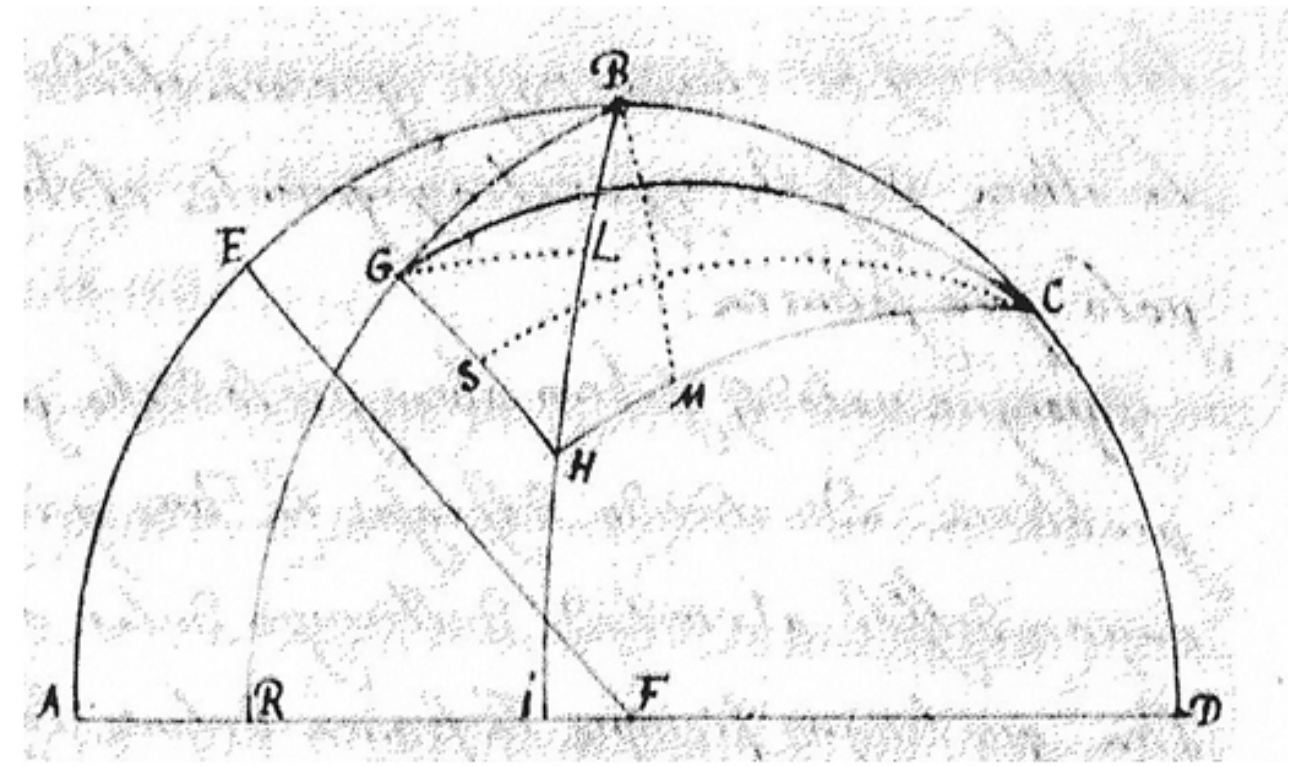




\section{NOTAS}

1 Reconocimiento de los estrechos de Magallanes y San Vicente, con algunas cosas curiosas de navegación (1621), Biblioteca Nacional, MS 3190.

2 Nos encontramos en un periodo en el que no se ha realizado la famosa transición del Arte de Navegar (basada en la experiencia y el conocimiento empírico) a la Navegación Científica, que trata de resolver problemas de navegación a partir de la Geometría.

3 El problema ya aparece resuelto en libros de Clavius, o Nunes, quien describió un método basado en el uso de una esfera y la medida de dos azimuts del Sol. Pero Diego Ramírez repasa todos estos métodos y establece otro que considera más exacto y ágil para el cálculo.

4 Esta carta náutica, que contaba con los más exactos datos de latitud y longitud de la época, fue utilizada por los pilotos españoles que durante el siglo XVII se adentraban por el estrecho de Magallanes.

5 Los pilotos mayores de la Casa de la Contratación de Sevilla en el siglo XVI evolucionaron en cuanto a su estilo de concebir la náutica (y las relaciones entre experiencia y teoría en cuestiones de navegación) desde Américo Vespucio, nombrado en 1508, Díaz de Solís, en 1512, Sebastián Caboto, en 1518; siendo los tres de un talante más bien empírico en su modo de entender la náutica; hasta Alonso de Chaves, nombrado en 1552, Zamorano, en 1586, Andrés García de Céspedes, en 1596, y de nuevo Zamorano en 1596 hasta su muerte, que sería sucedido por Diego Ramírez de Arellano. Los tres últimos pilotos mayores el siglo XVI tenían un talante más bien teórico de la náutica; y la transición de un tipo de Piloto Mayor a otro tipo no fue casual.

6 Para la actualización del padrón real por el procedimiento de cálculo de la longitud geográfica por medio de eclipses, véase Rodríguez Sala, María Luisa (1998), El eclipse de luna: misión científica de Felipe II en Nueva España, Huelva, Universidad de Huelva.

7 Es esclarecedora la carta de Bernardino de Mendoza, embajador en Londres, al rey, fechada en 10 de junio de 1579, del AGI, Patronato, 266, R 28. En torno al año 1579 proliferan los archivos con un contenido similar al de esta carta del embajador en Londres. Son o bien cartas del embajador o petición de informes del rey sobre la situación de hostilidad en Inglaterra hacia España.

8 Todos los métodos incluidos en este capítulo se basan en última instancia en el triángulo de posición formado por el Sol, el zenit y el polo, cuyo análisis por trigonometría esférica conduce a tres grupos de fórmulas, que relacionan las magnitudes a: altura del Sol, I: latitud, A: azimut, h: ángulo horario (equivalente a las horas desde que el Sol culmina, en sentido este-oeste, siendo 1hora = 15ㅇ), d: declinación solar. Según las magnitudes que conozcamos, la latitud se obtiene usando una de estas fórmulas válidas para el triángulo de posición: 1 ) sen $\mathrm{a}=\operatorname{sen} \mathrm{I} \operatorname{sen} \mathrm{d}+\cos$ I $\cos d \cos h$, sen $d=\operatorname{sen}|\operatorname{sen} a+\cos | \cos a \cos z, 2) \cos \mid /$ sen $a=\cos d / \operatorname{sen} z=\cos a / \operatorname{sen} h, 3) \operatorname{tg} a \cos I=\operatorname{sen} I \cos z+\operatorname{sen} z$ $\operatorname{ctg} h, \operatorname{tg} d \cos l=\operatorname{sen} I \cos h+\operatorname{sen} h$ ctg $z$. Por ejemplo, en caso de que conozcamos la altura solar a y la hora a la cual se realizó la observación $\mathrm{h}$, usamos la igualdad sen $\mathrm{a}=\operatorname{sen} \mathrm{I} \operatorname{sen} \mathrm{d}+\cos I \cos$ $\mathrm{d} \cos \mathrm{h}$, que nos permite deducir el valor de $\mathrm{l}$.

9 Este modo de averiguar la latitud del lugar es un caso en el que se utilizan alturas extrameridianas del Sol (de hecho, instantes de altura cero, con la inexactitud que conlleva la determinación de altura cero sin tener en cuenta la corrección por refracción, correcciones que se empezarían a tener en cuenta avanzado el siglo XVII) no supone exactamente el conocimiento de la altura del polo a cualquier hora del día.

10 Estrictamente la latitud se podría saber con la hora del orto o el ocaso en el lugar y la declinación de la fecha, pero proporcionaría un error considerable por el hecho ya comentado de ser muy inexacta la determinación de la hora con los relojes de la época.

11 El ángulo medido desde el sur que forma el vertical del astro sobre el horizonte del lugar.

12 Véase Albuquerque, Luis de (1975), Estudos de História, vol. III: A navegaçao astronómica; Coimbra, Acta Universitatis Conimbrigensis, pp. 107-127.

13 La proyección estereográfica consiste en proyectar la esfera terrestre sobre un plano tangente a la misma. El punto de tangencia determina el aspecto de la proyección. Si es un polo el punto de tangencia se tiene una proyección estereográfica polar; si es un punto del ecuador es una proyección estereográfica meridiana o ecuatorial; y si es un punto sobre la esfera diferente de los anteriores se denomina estereográfica horizontal u oblicua. En este caso, Céspedes describe una proyección meridiana o ecuatorial.

14 Una diferencia atribuible al hecho de que Diego Ramírez no tenía en cuenta las correcciones por refracción atmosférica a las alturas observadas.

15 Método que desarrolla Clavius en el libro III del Astrolabio, canon 13 número 2.

16 Es otro tópico el hecho de que la matemática griega eludió la faceta numérica por diversas razones, entre otras por la dificultad que presentaba el sistema de numeración griego y su falta de operatividad en el cálculo.

17 En terminología moderna, Diego Ramírez hace uso de los cuatro grupos de fórmulas que se deducen para cualquier triángulo esférico: 1) fórmulas que relacionan tres lados y un ángulo, del tipo $\cos a=\cos b \cos c+\operatorname{sen} b \operatorname{sen} c \cos A, 2)$ fórmulas que relacionan dos lados y sus ángulos opuestos, de tipo sen $A$ / sen $a=\operatorname{sen} B / \operatorname{sen} b=\operatorname{sen} C / \operatorname{sen} c, 3$ ) fórmulas que relacionan dos lados, el ángulo comprendido entre ambos y el opuesto a uno de ellos, del tipo $\operatorname{cotg} b \operatorname{sen} c=\cos c \cos A+\operatorname{sen} A \operatorname{cotg} B$; 4) fórmulas que relacionan tres ángulos y un lado, del tipo $\cos A=$ - $\cos B \cos C+\operatorname{sen} B$ sen $C \cos$ a. Diego Ramírez, en la descripción del método alude en cada paso al capítulo de los Esféricos de Clavius o de Theodosio donde se encuentra deducido el resultado que aplica en el proceso que está describiendo, que siempre es uno de los cuatro comentados anteriormente y expresados según la terminología moderna.

18 La proposición a que se refiere Diego Ramírez dice: Per duo puncta data in sphaerica superficie maximun circulum describere, que es simplemente la afirmación del hecho de que por dos puntos de la superficie de la esfera pasa un círculo máximo, que cumple la propiedad de ser la línea más corta entre esos dos puntos. Se encuentra en una obra de Clavius sobre Theodosio titulada Theodosii Sphaericorum.

19 Esta proposición 64 de los Triángulos esféricos de Clavius se encabeza del siguiente modo: Datis duobus arcubus trianguli sphaerici non rectanguli, cum angulo ab ipsis comprehenso, reliquum arcum, cum reliquis angulis reperire. En el desarrollo de la proposición se ha de dar un procedimiento y una regla para calcular, a partir de dos lados de un triángulo esférico y el ángulo entre ellos abarcado, el otro lado y los dos ángulos que 
faltan. Modernamente corresponde al uso de la fórmula cos $b$ $=\cos a \cos c+\operatorname{sen} a \operatorname{sen} c \cos A$. No obstante, Clavius deduce el modo de calcular los elementos que faltan en el triángulo esférico, dividiéndolo en triángulos esféricos rectángulos que ya ha estudiado en capítulos precedentes del libro.

20 Es un caso en el cual se conocen los tres lados del triángulo esférico, entonces se calculan los tres ángulos por medio de la fórmula $\cos A=(\cos a-\cos b \cos c) / \operatorname{sen} b$ sen $c$. En la proposición

\section{BIBLIOGRAFÍA}

Albuquerque, Luis de (1975), A navegaçao astronómica. Estudos de História, vol. III, Coimbra, Acta Universitatis Conimbrigensis.

Cerezo Martínez, Ricardo (1994), La Cartografía Náutica Española en los Siglos XIV, XV y XVI, Madrid, CSIC.

Díaz Hernández, Ignacio (2011), Estudio preliminar y transcripción del manuscrito de Diego Ramírez de Arellano "Reconocimiento de los estrechos de Magallanes y San Vicente, con algunas cosas curiosas de navegación" (1621), Valencia, Publicaciones de la Universitat de València, Tesis doctoral [CD-ROM].

Fernández de Navarrete, Martín (1846), Historia de la Náutica y las Ciencias Matemáticas, Madrid, Imprenta de la viuda de Calero.

González González, Francisco (1992), Astronomía y navegación en España, siglos XVI-XVIII, Madrid, Mapfre.

López Piñero, José María (1986), El arte de navegar en la España del renacimiento, Barcelona, Labor.

López Piñero, José María (2007), La tecnología de la navegación en la España renacentista, Valencia, Ayuntamiento de Valencia.

Martínez Hidalgo y Terán, José María (1946), Historia y leyenda de la aguja magnética, Barcelona, Gustavo Gili.

Navarro Brotons, Víctor (1998), Matemáticas, cosmología y humanismo en la España del siglo XVI: Los comentarios al segundo Libro de la Historia Natural de Plinio de Jerónimo Muñoz, Valencia, Instituto de Estudios Documentales e Históricos sobre la Ciencia, Universidad de Valencia.

Navarro Brotons, Victor y Salavert Fabiani, Vicente (2006), "El cultivo de la geografía y las relaciones entre la geografía y la cosmografía en la España Renacentista", En: Vicente Maroto, I. y Esteban Piñero, M. (coords.), La ciencia y el mar, Valladolid, Server-Cuesta Impresores, pp. 209-228.
63 de los Esféricos de Clavius encontramos el encabezamiento: Datis ómnibus trianguli sphaerici non rectanguli, omnes tres eius angulos investigare. Llega a dar Clavius una regla de cálculo, deducida a partir de la descomposición del triángulo inicial en triángulos esféricos rectángulos.

21 Se trata de un caso similar al comentado en una nota anterior, es decir, son conocidos dos lados del triángulo rectángulo y el ángulo comprendido entre ellos.

Pérez-Mallaina Bueno, Pablo Emilio (1996), El hombre frente a mar: Naufragios en la Carrera de Indias durante los siglos XVI y XVII, Sevilla, Universidad de Sevilla.

Portuondo, Maria (2005), Secret Science: Spanish Cosmography and the new world, Ph.D.dissertation, Maryland, The Johns Hopkins University.

Pulido Rubio, José (1950), El Piloto Mayor de la Casa de Contratación de Sevilla. Pilotos mayores, catedráticos de cosmografía y cosmógrafos, Sevilla, Escuela de estudios HispanoAmericanos, CSIC.

Rodríguez Sala, María Luisa (1998), El eclipse de luna: misión científica de Felipe II en Nueva España, Huelva, Servicio de Publicaciones de la Universidad de Huelva.

Sandman, Alison (2001), Cosmographers vs. Pilots: Navigation, Cosmography and the state en Early Modern Spain, Madison, University of Wisconsin.

Soler Pascual, Emilio (2010), Reconocimiento de los estrechos de Magallanes y de San Vicente con algunas cosas curiosas de navegación: por el capitán Diego Ramírez de Arellano, cosmógrafo y piloto mayor del Rey en la contratación de Sevilla, Valencia, Institución Alfonso el Magnánimo.

Vicente Maroto, Isabel (2001), "La expedición de los hermanos Nodal y del cosmógrafo Diego Ramírez de Arellano", Revista de Historia Naval, 83, pp. 7-28.

Vicente Maroto, Isabel y Esteban Piñeiro, Mariano (1991), Aspectos de la ciencia aplicada en la España del siglo de Oro, Salamanca, Junta de Castilla y León. 